\title{
THE ASYMPTOTIC SOLUTIONS OF ORDINARY LINEAR DIFFERENTIAL EQUATIONS OF THE SECOND ORDER, WITH SPECIAL REFER- ENCE TO THE STOKES PHENOMENON†
}

BY R. E. LANGER

1. Introduction. From the title of my address you will already have observed that it is my intention to discuss with you the asymptotic representation of the solutions of certain differential equations, and that my point in doing this is largely to dwell upon the Stokes phenomenon which arises in that theory. There are at least two good reasons why I have chosen to do this. Firstly, the subject seems to me to have mathematical features of distinct interest and perhaps of importance, and, secondly, it has risen during the last dozen years to a position of importance in theoretical physics, both of the more classical type and of the modern quantum mechanics. The instance is indeed one in which the development of mathematics has not merely been hastened by the spur of the needs of growing physical theory, but in which investigators primarily or at least prominently identified as physicists have turned aside to mathematical deductions to fill their own needs.

The Stokes phenomenon is named after its discoverer, the British mathematician and physicist, Sir G. G. Stokes. He was confronted by its manifestation in his study of the Bessel functions, and that it held puzzlement and difficulty for him he was not loath to acknowledge. With your permission I should, indeed, like to read you an abstract from a letter which he wrote at the time, as you will conclude, to a certain young lady: $\ddagger$ London, March 19/57. "When the cat's away the mice may play. You are the cat and I am the poor little mouse. I have been doing what I guess you won't let me do when we are married, sitting up till 3 o'clock in the morning fighting hard against a mathematical difficulty. Some years ago I attacked an integral of Airy's, and after a severe trial reduced it to a readily calculable form. But there was one difficulty about it which,

$\dagger$ A symposium lecture delivered by invitation of the program committee at the meeting of the Society at Chicago, April 6, 1934.

$\ddagger$ Sir George Gabriel Stokes, Memoirs and Scientific Correspondence, Cambridge, 1907, vol. 1, p. 62 . 
though I tried till I almost made myself ill, I could not get over, and at last $I$ had to give it up and profess myself unable to master it. I took it up again a few days ago, and after two or three days' fight, the last of which I sat up till 3, I at last mastered it. ..." A decade later the phenomenon was observed, again in connection with the Bessel functions, by Hankel, who speaks of his investigations as having led to "peculiar results which might perhaps serve as a basis for a general theory of semi-convergent series," those remarkable developments whose true nature was almost entirely unknown. $\dagger$

2. The Sturm-Liouville Equation. Let me, to begin with, write down the partial differential equation

$$
\frac{\partial}{\partial x}\left\{k(x) \frac{\partial T}{\partial x}\right\}-q(x) T=p(x) \frac{\partial T}{\partial t}
$$

which controls the temperature $T$ in the flow of heat along a slender rod in the direction $x$ with time $t$. The coefficients $k$, $p$, and $q$ are all essentially positive functions, determined by the physical characteristics of the rod and the surrounding medium. A familiar mode of procedure toward solving the equation is to substitute

$$
T=f(t) u(x),
$$

whereupon the equation decomposes into the form

$$
\frac{f^{\prime}(t)}{f(t)}=\frac{\frac{d}{d x}\left(k \frac{d u}{d x}\right)-q u}{p u} .
$$

In this the left member is a function of $t$ alone and the right member is a function of $x$ alone, and the equality therefore implies that each is independent of both $x$ and $t$, say is equal to a quantity $-\lambda^{2}$. I am not interested in the equation in $t$. The equation for the component $u(x)$ is, however, of the form

$$
\frac{d}{d x}\left\{k(x) \frac{d u}{d x}\right\}+\left\{\lambda^{2} p(x)-q(x)\right\} u=0,
$$

$\dagger$ H. Hankel, Die Cylinderfunktionen erster und zweiter Art, Mathematische Annalen, vol. 1 (1869), p. 467. 
in which $\lambda^{2}$ is independent of $x$, but may be regarded as a parameter variable in its own right. The equation (1) is known as the differential equation of the Sturm-Liouville type, in recognition of the fundamental work done in connection with it by Sturm and Liouville about a century ago. It arises in the mathematical formulation of classical physical problems of such great diversity as the flow of heat, the vibrations of strings and membranes, the oscillations of rods and beams, the diffusion of substances through each other, the distribution of electrical potentials and current densities, the turbulent motions of fluids, the tides, etc. Mathematically it is the type equation which includes as special cases the differential equations of such standard functions as the trigonometric functions, the Bessel functions, the Legendre functions, the polynomials of Hermite, Tchebycheff, and Laguerre, certain confluent hypergeometric functions, the functions of Mathieu, and many others. Much more recently it has come into renewed prominence among the physicists as a type of the Schrödinger wave equation in the theory of quantum mechanics.

It is natural under these circumstances that much study has been given to the equation in the course of a century, with the result that in its present form its theory includes among others such beautiful chapters as those on the oscillation and comparison of solutions, the boundary problems, the expansion of arbitrary functions in series of solutions, the asymptotic forms of the solutions for large complex values of the variable, and the asymptotic dependence of the solutions upon the parameter $\lambda$. It is to the last two of these, and more particularly to the last one, to which I shall confine my considerations.

If in the form (1) the coefficient $k(x)$ is not zero on a given interval, and admits a second derivative, a familiar change of dependent variable reduces the equation to the form

$$
u^{\prime \prime}+\left\{\lambda^{2} \phi^{2}(x)-\chi(x)\right\} u=0 .
$$

In this $\phi^{2}$ and $\lambda^{2}$ are written in place of $\phi$ and $\lambda$ only as a matter of convenience. There is no presumption that these quantities are positive; indeed, for many considerations they need not even be real. The form (2) has the advantage of great simplicity and will, therefore, be made the immediate basis of our discussion. 
To avoid a dissipation of time upon matters which are after all of rather a secondary interest, I shall assume henceforth that where the equation (2) is to be considered its coefficients are continuous and, in fact, differentiable to whatever order may be required. To keep the discussion within proper bounds I shall have to confine myself very largely to real values of $x$ and $\lambda$, and when doing so I shall suppose $\lambda$ positive and shall refer to the range of values which $x$ may take on as the interval $a \leqq x \leqq b$. It is to be understood, however, that unless the contrary is stated the possibilities $a=-\infty$, or $b=+\infty$, or both, are not excluded.

3. The Asymptotic Solutions when $\phi^{2}(x)$ is Bounded From Zero. If $\phi^{2}(x)$ is real and is bounded from zero on $(a, b)$, we may define the root $\phi(x)$ so that $\phi /|\phi|$ is either 1 or $i$ according as $\phi^{2}$ is positive or negative. The change of variables

$$
t=\frac{1}{\kappa} \int_{*}^{x}|\phi| d x, \quad v=|\phi(x)|^{1 / 2} u, \quad i \rho=\kappa \lambda\left\{\frac{\phi}{|\phi|}\right\},
$$

in which $\kappa$ and $*$ may be any constants with respect to $x$, reduces the differential equation to the simple form

$$
\frac{d^{2} v}{d t^{2}}-\left\{\rho^{2}+\omega_{1}(t)\right\} v=0,
$$

with the variable $t$ ranging over an interval $\left(t_{a}, t_{b}\right)$ and with

$$
\omega_{1}(t)=\frac{\kappa^{2}}{|\phi|^{2}}\left\{x+\frac{\phi^{\prime \prime}}{2 \phi}-\frac{3 \phi^{\prime 2}}{4 \phi^{2}}\right\} .
$$

Now when $|\rho|$ is very large the first of the two terms $\rho^{2}$ and $\omega_{1}(t)$ greatly overshadows the second and effectively minimizes its influence. Since in the complete absence of $\omega_{1}(t)$ the equation would be solved by the functions $e^{ \pm \rho t}$, the suggestion is evidently compelling that the equation as it stands may be solvable, at least approximately, by an expression

$$
V_{n}(t, \rho) \equiv e^{\rho t} S(t, \rho),
$$

with $S$ some polynomial in $1 / \rho$, say

$$
S(t, \rho) \equiv 1+\frac{\alpha_{1}(t)}{\rho}+\frac{\alpha_{2}(t)}{\rho^{2}}+\cdots+\frac{\alpha_{n}(t)}{\rho^{n}} .
$$


This expression is found directly to satisfy a differential equation

$$
V_{n}^{\prime \prime}-\left\{\rho^{2}+\omega_{1}(t)+\frac{\Theta}{S}\right\} V_{n}=0,
$$

in which $\Theta$ is explicitly the sum of terms

$$
\Theta \equiv\left(2 \alpha_{1}^{\prime}-\omega_{1}\right)+\sum_{j=2}^{n}\left(\frac{2 \alpha_{j}^{\prime}-\alpha_{j-1} \omega_{1}+\alpha_{j-1}^{\prime \prime}}{\rho^{j-1}}\right)+\left(\frac{\alpha_{n}^{\prime \prime}-\alpha_{n} \omega_{1}}{\rho^{n}}\right),
$$

and this conclusion follows irrespective of what the coefficients $\alpha_{j}(t)$ may be. It is at once clear, however, that with such coefficients as fulfill the relations

$$
\alpha_{1}^{\prime}=\frac{1}{2} \omega_{1}, \quad \alpha_{j}^{\prime}=\frac{1}{2}\left(\alpha_{j-1} \omega_{1}-\alpha_{j-1}^{\prime \prime}\right), \quad(j=2,3, \cdots, n),
$$

all terms of $\Theta$ but the last vanish. Now the successive determination of functions $\alpha_{j}(t)$ which do satisfy the relations (4) involves at each step at most a quadratureand is therefore a matter of no theoretical difficulty, at least if $\omega_{1}(t)$ is absolutely integrable over $\left(t_{a}, t_{b}\right)$. This condition is surely fulfilled if the interval is finite. It is seen thus how a form $V_{n}$ may be constructed to solve a differential equation

$$
V_{n}^{\prime \prime}-\left\{\rho^{2}+\omega_{1}(t)+\frac{\alpha_{n}^{\prime \prime}-\alpha_{n} \omega_{1}}{S \rho^{n}}\right\} V_{n}=0,
$$

and since this equation resembles the equation (3) and does so the more closely the larger $n$ and $|\rho|$ are taken, the presumption becomes strong that, with $V_{n}(t, \rho)$ determined as indicated, the latter is an asymptotic representation of a true solution of the equation (3). Inasmuch as this latter equation depends only on $\rho^{2}$, the same might naturally be expected also of the form $V_{n}(t,-\rho)$. With proper adjustments these facts can indeed be shown. $\dagger$ I shall, however, omit a proof at this point, particu-

$\dagger \mathrm{J}$. Horn, Ueber eine lineare Differentialgleichung zweiter Ordnung mit einem willkürlichen Parameter, Mathematische Annalen, vol. 52 (1899), p. 271, and other papers by this author of about this date. G. D. Birkhoff, On the asymptotic character of the solutions of certain linear differential equations containing a parameter, Transactions of this Society, vol. 9 (1908), p. 219. O. Blumenthal, Ueber asymptotische Integration linearer Differentialgleichungen, etc., Archiv der Mathematik und Physik, vol. 19 (1912), p. 136. 
larly as $\mathbf{l}$ shall later sketch out considerations in which such a proof is effectively included.

The facts to be noted are that under the conditions assumed a pair of solutions $v_{ \pm}(t)$ of the equation (3) exist which fulfill a set of relations

$$
\left|v_{ \pm}(t)-V_{n}(t, \pm \rho)\right|<\frac{K}{\rho^{n+1}}\left|e^{ \pm \rho t}\right|
$$

with $K$ some constant and $t$ ranging over the interval $\left(t_{a}, t_{b}\right)$, or, to summarize in terms of the original variables:

If $\phi^{2}(x)$ is bounded from zero on $(a, b)$ and the integral

$$
\int_{a}^{b}\left|\frac{\chi}{\phi}+\frac{\phi^{\prime \prime}}{2 \phi^{2}}-\frac{3 \phi^{\prime 2}}{4 \phi^{3}}\right| d x
$$

converges, then the equation (2) has a pair of solutions $u_{ \pm}(x)$, which over the interval $(a, b)$ have the forms

$$
\begin{aligned}
u_{ \pm}(x)=\left.\frac{1}{|\phi| 1 / 2} e^{ \pm \lambda i}\right|_{* \phi d x} ^{x}\{1+ & \frac{\beta_{1}(x)}{\kappa \lambda}+\frac{\beta_{2}(x)}{(\kappa \lambda)^{2}} \\
& \left.+\cdots+\frac{\beta_{n}(x)+\epsilon_{ \pm}(x, \lambda)}{(\kappa \lambda)^{n+1}}\right\} .
\end{aligned}
$$

When $\phi^{2}<0$ on $(a, b)$, the forms (6), with $n=1$, show that there are solutions representable by the formulas

$$
u_{ \pm}(x)=\frac{1}{|\phi|^{1 / 2}} e^{ \pm \lambda \int_{*|\phi| d x}^{x}}\{1+O(1 /(\kappa \lambda))\} .
$$

On the other hand, if $\phi^{2}>0$, a suitable combination of these forms yields the representation

$$
u_{\gamma}(x)=\frac{1}{\phi^{1 / 2}}\left\{\cos \left(\lambda \int_{*}^{x} \phi d x+\gamma\right)+O(1 /(\kappa \lambda))\right\},
$$

in which $\gamma$ may be any constant. The symbol $O(1 /(\kappa \lambda))$ stands in each case for a function of $x$ and $\lambda$ which on $(a, b)$ is uniformly of the order of $1 /(\kappa \lambda)$ when $\kappa \lambda$ is sufficiently large.

I have supposed in this statement and throughout that $\lambda$ has been taken real and positive. The formal work needs no modification when it is thought of as complex. The proof, how- 
ever, requires that the quantity $\lambda i \int_{*}^{x} \phi d x$ remain in either a right or a left hand half of the complex plane. A representation (6) is, to be sure, obtained in either case, but the question of the relation between the solutions $u$ which are involved in the two cases is left open.

I need perhaps hardly remark that once the forms (6) are known the change of variables used above need no longer be taken into account. The coefficients $\beta_{j}(x)$ may be successively determined by substitution of the forms directly into the equation (2).

It may at this point be appropriate for me to outline also a variation of the procedure above. You will, however, recognize it as of only formal and minor significance. If a solution of the equation (2) is assumed in the form

$$
u(x)=e^{\lambda \int_{* \sigma(x, \lambda) d x}^{x},}
$$

there results for $\sigma$ the Riccatti equation

$$
\frac{\sigma^{\prime}}{\lambda}+\sigma^{2}+\phi^{2}-\frac{\chi}{\lambda^{2}}=0,
$$

and if $\sigma$ is taken as a series in $1 / \lambda$, that is,

$$
\sigma(x, \lambda)=\sum_{j=0}^{\infty} \frac{\sigma_{j}(x)}{\lambda^{i}},
$$

the differential equation becomes

$$
\begin{gathered}
\left(\sigma_{0}^{2}+\phi^{2}\right)+\frac{1}{\lambda}\left(2 \sigma_{1} \sigma_{0}+\sigma_{0}^{\prime}\right)+\frac{1}{\lambda^{2}}\left(2 \sigma_{2} \sigma_{0}+\sigma_{1}^{2}+\sigma_{1}^{\prime}-\chi\right) \\
+\sum_{j=3}^{\infty} \frac{1}{\lambda^{j}}\left(\sigma_{j-1}^{\prime}+\sum_{n=0}^{j} \sigma_{n} \sigma_{n-j}\right)=0 .
\end{gathered}
$$

By choosing the coefficients $\sigma_{j}(x)$ so as to make the individual terms zero, that is,

$$
\begin{array}{ll}
\sigma_{0}= \pm i \phi, & \sigma_{2}=-\frac{1}{2 \sigma_{0}}\left(\sigma_{1}^{\prime}+\sigma_{1}^{2}-\chi\right), \\
\sigma_{1}=-\frac{1}{2 \sigma_{0}} \sigma_{0}^{\prime}, & \sigma_{j}=-\frac{1}{2 \sigma_{0}}\left(\sigma_{j-1}^{\prime}+\sum_{n=1}^{j-1} \sigma_{n} \sigma_{j-n}\right),
\end{array}
$$


where $j=3,4, \cdots$, the equation is formally solved. This form of procedure $\dagger$ is in some vogue among the physicists, to whom it is known as part of the so called "Wentzel, Kramers, Brillouin," (W.K.B.), method. I shall have occasion to refer to this further.

The formulas (6) each include a function which has been designated by $\epsilon$, and which is not specified beyond the statement that it approaches zero as $\kappa \lambda$ becomes infinite. The presence of these terms represents, therefore, a certain degree of vagueness in the formulas. This is a general characteristic of asymptotic representations and is not to be neglected, for to do so may easily lead into an utter misuse of the formulas. In most actual cases more precise analysis may be made to yield specific bounds for the magnitudes of the $\epsilon$ terms. With such bounds at hand and with a definite margin of permissible error given, the condition that the vagueness fall within the allowed margin must be looked upon as fixing a lower bound, say $N$, to the values of $k \lambda$ for which the formulas may be used. In any application of them it must then be regarded as a tacit hypothesis that $|\kappa \lambda| \geqq N$.

4. Consideration of a Point where $\phi^{2}(x)$ is Zero. Let us suppose now that at a point of the $x$ axis, say at $x_{0}$, the function $\phi^{2}(x)$ becomes zero, and to fix the ideas let us suppose that the zero is a simple one and that $\phi^{2}$ changes from negative to positive with increasing $x$. Then in any fixed closed interval on the left of but not including $x_{0}$ there are solutions represented by the forms (7a) when $\kappa \lambda$ is large. Every solution is therefore represented by some linear combination of these forms, with coefficients which are free from $x$, and so the solutions are to be characterized as of an exponential type. On the other hand, in any closed fixed interval on the right of and not including $x_{0}$, every solution is represented by a formula ( $7 \mathrm{~b})$ and so is of an oscillatory type. I wish for a moment to focus the attention upon such an interval, let us say for definiteness one on the right of $x_{0}$, with a view toward determining how large the interval $\left(x_{0}, a\right)$ must be in order that the forms may be valid for $x \geqq a$.

The deductions of $\$ 3$ were built upon the assumption that

$\dagger$ G. Wentzel, Eine Verallgemeinerung der Quantenbedingungen für die Zwecke der Wellenmechanik, Zeitschrift für Physik, vol. 38 (1926), p. 518. 
$|\rho|$ was sufficiently large to bring the vagueness of the resulting forms within the admissible bounds of error. The symbols $*$ and $\kappa$ remained unspecified except that they were to be constant. Let us choose these values now as

$$
*=x_{0}, \quad \kappa=\int_{x_{0}}^{a}|\phi| d x .
$$

The point $t_{a}$ then remains fixed independently of the value $a$, the reasoning used in $\$ 3$ applies, and the forms (6) are deducible. Thus the condition under which these forms suitably represent solutions is that $\lambda \int_{x_{0}}^{a}|\phi| d x$ be sufficiently large, and this clearly permits the point $a$ to be taken closer and closer to $x_{0}$ as $\lambda$ is taken larger and larger. Since the value of $\xi$, where

$$
\xi=\lambda \int_{x_{0}}^{x} \phi d x,
$$

increases numerically as $x$ recedes in either direction from $x_{0}$, it follows that the forms (6) or (7) are usable in intervals in which $|\xi| \geqq N$. I have, of course, assumed here that the intervals are ones on which $\phi^{2}$ is bounded from zero except near $x_{0}$.

With a value of $N$ prescribed, the relation $|\xi|=N$ defines two points, say $x_{-N}<x_{0}$ and $x_{N}>x_{0}$. As we have seen, these points depend upon $\lambda$ and approach $x_{0}$ as $1 / \lambda$ approaches zero. The interval $\left(x_{-N}, x_{N}\right)$ thus separates any one in which a solution is described by a combination of the forms (7a) from one in which it is of the form (7b), and hence in this interval the transition of a solution from the exponential to the oscillatory type takes place. That no information as to the mode of this transition is likely to be obtainable from the forms (7) themselves becomes immediately evident when it is observed that without exception these forms become infinite as $x_{0}$ is approached. The differential equation satisfied by their leading terms is found directly to be

$$
U^{\prime \prime}(x)+\left\{\lambda^{2} \phi^{2}+\frac{\phi^{\prime \prime}}{2 \phi}-\frac{3 \phi^{\prime 2}}{4 \phi^{2}}\right\} U=0,
$$

and this, unlike the equation (2), has a singularity at the point $x_{0}$. The interval $\left(x_{-N}, x_{N}\right)$ is, therefore, evidently one in which no actual resemblance between the differential equations exists to be built upon. 
It might still seem conceivable that by a passage through complex values encircling the point $x_{0}$ the representation valid for $x \geqq x_{N}$ might be found to serve also for $x \leqq x_{-N}$. Such expectations are shaken, however, when it is observed that if $\phi^{2}$ is analytic the same is true of the solutions $u(x)$, whereas the forms (6) fail even to be single-valued in the region about $x_{0}$.

5. The Stokes Phenomenon in the Theory of the Bessel Functions. The differential equation for the Bessel functions $J_{\nu}(\xi)$, considered in the complex $\xi$ plane, has two singular points located respectively at $\xi=0$ and $\xi=\infty$. For large values of $|\xi|$ asymptotic forms for these functions have been deduced, generally through the medium of contour integral solutions of the equation, and when $\arg \xi$ is fixed, the functions are accordingly known to be represented by formulas

$$
J_{ \pm \nu}(\xi) \sim \frac{1}{(2 \pi \xi)^{1 / 2}}\left\{C_{ \pm 1} e^{i \xi} P\left(\frac{1}{\xi}\right)+C_{ \pm 2} e^{-i \xi} P\left(\frac{-1}{\xi}\right)\right\},
$$

where $P$ denotes a formal power series with initial term 1 , and the coefficients are appropriate constants. $\dagger$

Now if the constants $C$ are determined so that the formulas are correct, let us say for real positive values of $\xi$, then they will in general not be correct when $\xi$ is negative. More generally speaking, if $\arg \xi$ is allowed to vary, say to increase steadily, then it is found that ever and anon the formulas theretofore valid cease to remain so, and that to retain them, the coefficients $C_{ \pm 1}$ or $C_{ \pm 2}$ must be changed. This is the Stokes phenomenon, and, as I have already indicated, its initial comprehension was not without difficulty. The functions on the left of formulas (11) are after all continuous in $\arg \xi$, whereas any change of a coefficient upon the right has every appearance of a discontinuous change in that member of the relation.

The explanation of the anomaly is nevertheless simple. To begin with, it may be observed that when $|\xi|$ is large the numerical value of $e^{i \xi}$ varies alternately between the large and the very small as $\arg \xi$ continues to increase. The dominance in the expression (11), therefore, passes alternately from the one term to the other. From here on I cannot do better than to quote directly from Stokes to the following effect: "The way in

$\dagger$ †. N. Watson, A Treatise on the Theory of Bessel Functions, 1922, p. 202. 
which the paradox $\cdots$ is explained is this. A semiconvergent series (considered numerically, and apart from its analytic form) defines a function only subject to a certain amount of vagueness which is so much the smaller as the modulus of the variable $\cdots$ is larger. ‥ For critical values of $\theta$ [that is, $\arg \xi]$ the vagueness of the superior function becomes sufficient to swallow up the inferior function. As $\theta$ passes through the critical value the inferior term enters as it were into a mist, is hidden for a little from view, and comes out with its coefficient changed."

It is evident at once that the first term in formulas (11) overshadows the second in the lower half-plane, and that the converse is true in the upper half-plane. We may think, therefore, of certain lines extending from the origin to $\infty$, one in each upper and lower half-plane, in crossing which the coefficients are to be changed. I shall refer to them as Stokes' lines. The precise location of such a line is never a matter of importance, since the term which is overshadowed remains so in a sector of which the axis of imaginaries is the bisector and which may be anything definitely less than $\pi$ in magnitude.

Finally, I shall record for subsequent use two sets of constants which are appropriate to the formulas (11) when $\nu=1 / 3$, namely,

$$
\begin{aligned}
& C_{ \pm 1}=e^{\pi i(-1 / 4 \mp 1 / 6)}, \quad C_{ \pm 2}=e^{\pi i(1 / 4 \pm 1 / 6)}, \quad(-\pi<\arg \xi<\pi) \\
& C_{ \pm 1}=e^{\pi i(-1 / 4 \mp 1 / 2)}, \quad C_{ \pm 2}=e^{\pi i(1 / 4 \pm 1 / 6)}, \quad(0<\arg \xi<2 \pi) .
\end{aligned}
$$

6. A Particular Differential Equation. Perhaps the simplest equation of the type (2) in which $\phi^{2}(x)$ has a zero, is the differential equation

$$
y^{\prime \prime}+\lambda^{2} x y=0 .
$$

It is explicitly solved by the functions

$$
y_{ \pm}(x)=x^{1 / 2} J_{ \pm 1 / 3}(\xi), \quad \text { with } \quad \xi=\frac{2}{3} \lambda x^{3 / 2} .
$$

Its study, because of this, involves a minimum of difficulties with a maximum of explicitness, which accounts largely for the fact that the equation has come to play a rather central role in the general theory. I shall devote the present section to a discussion of it. 
If $\eta$ is constant with respect to $x$, and is arbitrary except that it is restricted to the range $-\pi / 2<\eta \leqq \pi / 2$, the formula

$$
\begin{aligned}
y_{\eta}(x)= & \Psi \lambda^{1 / 6}\left(\frac{2 \pi}{3}\right)^{1 / 2} \xi^{1 / 3}\left\{\cos \left(\frac{\pi}{3}-\eta\right) J_{-1 / 3}(\xi)\right. \\
& \left.+\cos \left(\frac{\pi}{3}+\eta\right) J_{1 / 3}(\xi)\right\},
\end{aligned}
$$

in which $\Psi=(2 / 3)^{1 / 6}$, gives, except for a constant factor, the general solution of the differential equation. When $x$ is real and positive the same is true of $\xi$; while negative values of $x$, say $\arg x=\pi$, yield values of $\xi$ for which $\arg \xi=3 \pi / 2$, namely, $i \xi$ $=|\xi|$. The first and second of the sets of coefficients (11a) are, therefore, to be associated respectively with the positive and negative values of $x$ in the applications of the formulas (11). In the case at hand, that is, of equation (12), the point $x_{0}$ is the origin and the points at which $|\xi|=N$ are $x_{ \pm N}=(3 N /(2 \lambda))^{2 / 3}$.

From the formulas (11), the asymptotic representations of $y_{\eta}(x)$ are found to be as follows: when $x \leqq x_{-N}$,

$$
\begin{aligned}
y_{\eta}(x)= & \frac{1}{|x|^{1 / 4}}\left\{\sin \eta e^{|\xi|}\left\{1+O\left(\frac{1}{|\xi|}\right)\right\}\right. \\
& \left.+\frac{\cos \eta}{2} e^{-|\xi|}\left\{1+O\left(\frac{1}{|\xi|}\right)\right\}\right\},
\end{aligned}
$$

and when $x \geqq x_{N}$,

$$
y_{\eta}(x)=\frac{1}{x^{1 / 4}}\left\{\cos \left(\xi-\frac{\pi}{4}+\eta\right)+O\left(\frac{1}{\xi}\right)\right\},
$$

and these involve a number of features which are worth noting. To begin with, it is clear from (14a) that as $x \rightarrow-\infty$, and hence $|\xi| \rightarrow+\infty$, the solutions with just one exception become infinite. The exceptional one is that for which $\eta=0$, that is, $y_{0}(x)$, and this approaches the limit zero. It is the only solution which is bounded for all values of $x$.

Like all asymptotic representations the formulas (14) involve an element of vagueness in the presence of the terms designated by $O(1 / \xi)$. These terms are largest at the point $x_{-N}$ or $x_{N}$, as the case may be, and if the formulas are to be taken as specific or 
explicit to a high degree, and this is indeed a tacit assumption when only the leading terms are explicitly written down, then it may be inferred that $N$ is large and the terms $O(1 / \xi)$ so small whenever $|\xi| \geqq N$ that they are for most purposes negligible.

Let us consider the case in which $\eta \neq 0$ and is such that at some point $x_{-T}$, where $x_{-T}<x_{-N}$, the relation

$$
\tan \eta=\frac{1}{2} e^{-2 \mid \xi-} T(x) \mid
$$

is fulfilled. Then at this point the explicit parts of the two terms in formula (14a) are equal. As $x$ moves thence to the right, the second term rises rapidly to dominance and its margin of vagueness soon swallows up the first term entirely. As $x$ moves to the left, on the other hand, the second term rapidly diminishes and is soon submerged in the margin of vagueness of the dominant first term. With the possible exception of the immediate neighborhood of the point $x_{-T}$, the graph of the solution accordingly falls when $x<x_{-T}$, and rises on the interval $x_{-T}<x \leqq x_{-N}$. The subscript $T$ was chosen to suggest $x_{-T}$ as such a point of transition.

An interval $\left(x_{-T}, x_{-N}\right)$ evidently exists only if $\eta$ is small enough to satisfy a relation (15), that is, if $\eta<\eta_{0}$, where

$$
\tan \eta_{0}=\frac{1}{2} e^{-2 N} \text {. }
$$

If we agree to think of $x_{-T}$ as coincident with $x_{-N}$ when $\eta \geqq \eta_{0}$, and likewise to think of $x_{-T}$ as $-\infty$ when $\eta=0$, the description of the solutions given in (14a) and (14b) may be recast into the form

$$
\begin{aligned}
& \text { (16a) } y_{\eta}(x)=\frac{\sin \eta}{|\phi|^{1 / 2}} e^{|\xi|}\{1+O(1 /|\xi|)\}, \text { when } x<x_{-T}, \\
& \text { (16b) } y_{\eta}(x)=\frac{\cos \eta}{2|\phi|^{1 / 2}} e^{-|\xi|}\{1+O(1 /|\xi|)\}, \\
& \text { when } x_{-T}<x \leqq x_{-N}, \\
& \text { (16 c) } y_{\eta}(x)=\frac{\left\{1+O\left(1 / \xi^{2}\right)\right\}}{\phi^{1 / 2}} \cos \left(\xi-\frac{\pi}{4}+\eta+O(1 / \xi)\right), \\
& \text { when } x \geqq x_{N} .
\end{aligned}
$$


The formula (16c) is readily identified as equivalent to (14b). I have written $\phi^{1 / 2}$ in place of $x^{1 / 4}$, inasmuch as the equation (12) is of the type (2) with $\phi^{2}=x$.

It is of some interest to note the inferences which can be drawn, especially with regard to the phase constant $\eta$, from the mere existence of representations identifiable with the ones thus given.

INFERENCE 1. If a solution $y(x)$ is of the form (16b) for all $x \leqq x_{-N}$, then $\eta=0$.

INFERENCE 2. If a solution $y(x)$ is of the form (16b) in some interval adjacent to $x_{-N}$, then $\eta<\eta_{0}$.

INFERENCE 3. If a solution $y(x)$ is of the form (16a) for $x \leqq x_{-N}$, then $\eta \geqq \eta_{0}$.

INFERENCE 4 . Since by taking $x$ sufficiently large the margin of vagueness of formula (16c) may be reduced to any degree, a representation (16c) determines $\eta$ and therefore the appropriate corresponding representations (16a) and (16b).

The differential equation

$$
y^{\prime \prime}+\lambda^{2} x^{\nu} y=0,
$$

which is more general than (12), is likewise explicitly solvable, namely, by the functions

$$
\text { (17a) } y_{ \pm}=x^{1 / 2} J_{ \pm 1 /(\nu+2)}(\xi), \quad \text { with } \quad \xi=\frac{2}{\nu+2} \lambda x^{(\nu+2) / 2}
$$

A discussion of it proceeds in a manner similar to that of equation (12) which has been given, and may be made the basis of the deductions concerning the general equation (2) when $\phi^{2}(x)$ becomes zero to the degree $\nu$. The index $\nu$ need not be an integer, but may be any real non-negative constant. In particular, the case $\nu=0$ is that in which $\phi^{2}$ is always of the same sign.

7. The Method of Jeffreys and Kramers. The first attack upon the equation (2) with $x$ ranging over an interval upon which $\phi^{2}(x)$ has a zero was made, so far as I know, by Jeffreys in $1923 . \dagger$

$\dagger \mathrm{H}$. Jeff reys, On certain approximate solutions of linear differential equations of the second order, Proceedings of the London Mathematical Society, (2), vol. 23 (1923), p. 428. 
He was led to it in an investigation of the oscillations of surfaces of water, his particular problem leading to the Mathieu equation

$$
u^{\prime \prime}+\left(R-2 h^{2} \cos 2 x\right) u=0
$$

When $|R|<2 h^{2}$, and $x$ is on a suitable interval, this is evidently an equation of the type(2). I shall sketch out Jeffreys' procedure, which he restricts to the case in which the zero of $\phi^{2}(x)$ is simple and in which $\chi(x) \equiv 0$, that is, to the equation

$$
u^{\prime \prime}+\lambda^{2} \phi^{2}(x) u=0 .
$$

There is no objection to taking the origin at the zero of $\phi^{2}$, nor to supposing that the positive $x$ direction is chosen so that $\phi^{2}$ has always the same sign as $x$. Finally, the slope of $\phi^{2}(x)$ at the origin may be taken as unity since this merely fixes the specification of $\lambda^{2}$. It is to be made a hypothesis now that in a neighborhood of the origin the function $\phi^{2}(x)$ is represented with sufficient accuracy by a linear function. With the normalization agreed upon above this linear function will, of course, be $x$ itself, and for small values of $x$ we have $\xi=\lambda \int_{0}^{x} \phi(x) d x=(2 / 3) \lambda x^{3 / 2}$, approximately. At the same time the differential equation (2a) is approximated by the equation (12) of $\$ 6$, namely,

$$
y^{\prime \prime}+\lambda^{2} x y=0
$$

Now when $x$ is negative and sufficiently large the solutions of both the equations (2a) and (12) are asymptotically representable, as is shown by the formulas (7a) and (16). We are led, therefore, to write

$$
\begin{aligned}
& u_{-}(x) \sim \frac{1}{|\phi|^{1 / 2}} e^{-|\xi|} \overline{\overline{\text { approx }}} \frac{1}{|x|^{1 / 4}} e^{-(2 / 3) \lambda|x| 3 / 2} \sim 2 y_{0}(x), \\
& u_{+}(x) \sim \frac{1}{|\phi|^{1 / 2}} e^{|\xi|} \overline{\overline{\text { approx }}} \frac{1}{|x|^{1 / 4}} e^{(2 / 3) \lambda|x| 3 / 2} \sim 2 y_{\pi / 6}(x) .
\end{aligned}
$$

On the other hand, when the origin has been traversed and $x$ is sufficiently removed therefrom on the positive side, the formulas (16c) and (7b) lead to 


$$
\begin{aligned}
2 y_{0}(x) & \sim \frac{2}{x^{1 / 4}} \cos \left(\frac{2}{3} \lambda x^{3 / 2}-\frac{\pi}{4}\right) \\
& =\frac{2}{\operatorname{approx}} \frac{2}{\phi^{1 / 2}} \cos \left(\xi-\frac{\pi}{4}\right) \sim 2 u_{\pi / 4}(x),
\end{aligned}
$$

$$
\begin{aligned}
2 y_{\pi / 6}(x) \sim & \frac{2}{x^{1 / 4}} \cos \left(\frac{2}{3} \lambda x^{3 / 2}-\frac{\pi}{12}\right) \\
& \overline{\overline{\text { approx }}} \frac{2}{\phi^{1 / 2}} \cos \left(\xi-\frac{\pi}{12}\right) \sim 2 u_{\pi / 12}(x) .
\end{aligned}
$$

The sequence of relations (18a) through (18b) is taken to supply the linear connections between the solutions of the formulas (7a) and (7b). These connections Jeffreys indicates in the form

$$
\begin{aligned}
& \text { (a) } \frac{1}{|\phi|^{1 / 2}} e^{-|\xi|} \longleftrightarrow \frac{2}{\phi^{1 / 2}} \cos \left(\xi-\frac{\pi}{4}\right) \\
& \text { (b) } \frac{1}{|\phi|^{1 / 2}} e^{|\xi|} \longleftrightarrow \frac{2}{\phi^{1 / 2}} \cos \left(\xi-\frac{\pi}{12}\right) \dagger,
\end{aligned}
$$

where, to quote him: "the sign $\longleftrightarrow$ is used to indicate that the functions it connects are asymptotic approximations to the same function in different circumstances."

The method seems to me to be very simple and ingenious. There are, however, a number of points involved in it which will bear discussion. To begin with, the proper use of the symbol $\sim$ is inevitably accompanied by the tacit assumption that $x$ is sufficiently removed from the origin, that is, to resort to our earlier notation, that $x \leqq x_{-N}$ or $x \geqq x_{N}$. The interval to be shunned, namely $\left(x_{-N}, x_{N}\right)$, depends in length upon the magnitude of the permitted margin of vagueness in the representations, and is to be shortened only at the expense of precision. As against this, the proper use of the symbol $\overline{\overline{\text { approx }}}$ is subject to the contingency that $|x|$ is sufficiently small, let us say for definiteness that $x_{-k} \leqq x \leqq x_{k}$. The length of this interval is limited by the hypothesis that within it the difference between $\phi^{2}(x)$ and the linear approximating function be sufficiently small to keep

$\dagger$ Through an evident slip Jeffreys obtained on the right of this formula the incorrect form $\left(2 / \phi^{1 / 2}\right) \cos (\xi-5 \pi / 12)$. 
under a permissible bound the cumulative error involved in the transfer from the solutions $u(x)$ to the solutions $y(x)$, and in the interchange between $\phi^{2}$ and $x$, and between $\xi$ and $(2 / 3) \lambda x^{3 / 2}$.

It is evidently a requirement of the method that the second of these intervals be large enough to contain the first within its interior, so that there may exist sub-intervals $\left(x_{-k}, x_{-N}\right)$ and $\left(x_{N}, x_{k}\right)$ in which the transfers may be made. It is not difficult to recognize that such an adjustment is always attainable by taking $\lambda$ suitably large if no restriction upon the magnitude of that parameter exists.

Jeffreys appraises the method in the terms "... it has the advantage of rapid application in general and of practicability in many cases where no definite integral solutions (of the differential equation) can be found. On the other hand, it is not usually easy to fix rigorously the limits of error involved in adopting it." This seems to me to touch upon both the strength and the weakness of the procedure. As to the approximate magnitude of the error, Jeffreys concludes that it decreases with increasing $\lambda$ and is of the order $O\left(\lambda^{-2 / 5}\right)$. I must, however, confess myself unable to follow him to my own satisfaction in this final part of his deduction.

Concerning the form of the statement of results (19) I would object to the use of the symbol $\longleftrightarrow$, on the ground that it invites misconceptions which have not failed to show themselves in the literature. I find it not unnatural to read into a pair of forms connected by an arrow the thought that in the direction of the arrow the one form implies the other. In the case of the relations (19) this inference would certainly be incorrect. A review of the specific implications which were drawn from the analogous formulas (16), and which were listed in $\$ 6$, will show the following, admitting that the formulas (19) ignore quantities of the order $O(1 / \xi)$ and $O(1 / \lambda)$.

Firstly, the relation (19a) is one in which the left hand member implies and necessarily leads to the right hand member. The converse, however, is not true, for it may be observed that in the deduction of the relation in question any solution $y_{\eta}(x)$ with $\eta$ of a suitable smallness, may replace the solution $y_{0}(x)$ used, while such solutions are not all associated with the form of the left hand member given.

Secondly, the relation $(19 \mathrm{~b})$ is one in which the right hand 
member implies and necessarily leads to the left hand member, but not conversely.

Three years subsequent to Jeffreys, that is, in 1926, Kramers, $\dagger$ in a discussion of the Schrödinger wave equation for a particle with one degree of freedom, encountered and again discussed the equation of the type (2a). His discussion is in every essential feature identical with that of Jeffreys and results in the deduction of the formula (19a). A subsequent paper $\ddagger$ adds to it the relation

$$
\frac{1}{|\phi|^{1 / 2}} e^{|\xi|} \longleftrightarrow \frac{1}{\phi^{1 / 2}} \cos \left(\xi+\frac{\pi}{4}\right),
$$

which is evidently the analog of the formula (16) in the particular case $\eta=\pi / 2$. There is, of course, no contradiction between the relations (19b) and (19c), since, as I have just remarked regarding the implication of one form from the other, the left hand form may be inferred from the right hand one in each case, but not conversely. Among the physicists these deductions, and especially the formulas (19a) and (19c), are known as part of the "W.K.B." (Wentzel, Kramers, Brillouin) method, which I have already mentioned in an earlier connection in $\$ 3$.

The extension of this method of procedure to the discussion of the differential equation of type (2a) in which $\phi^{2}(x)$ has a zero of any integral order was made by Goldstein $\S$ in 1927. He relates his paper directly to that of Jeffreys and has similar applications in mind.

8. The Method of Zwaan.\| A second method for dealing with the equation (2a) in the case that the zero of $\phi^{2}$ is a simple one is built upon the idea of avoiding the critical point at which $\phi^{2}$ vanishes, by encircling it in a passage through the complex plane. The procedure is the following, it being assumed by way

$\dagger$ H. A. Kramers, Wellenmechanik und halbzählige Quantisierung, Zeitschrift für Physik, vol. 39 (1926), p. 828.

$\ddagger$ H. A. Kramers, and G. P. Ittmann, Zur Quanteilung des asymmetrischen Kreisels II, Zeitschrift für Physik, vol. 58 (1929), p. 217.

$\S \mathrm{S}$. Goldstein, $A$ note on certain approximate solutions of linear differential equations of the second order, etc., Proceedings of the London Mathematical Society, (2), vol. 28 (1928), p. 81.

\| A. Zwaan, Intensitäten im Ca-Funkenspektrum, Dissertation, Utrecht, 1929. 
of an explicit hypothesis, that within a suitable region of the complex plane about the zero of $\phi^{2}$, the function $\phi^{2}(x)$ admits of approximation to the required degree of precision by means of a function which is analytic. For convenience I shall again suppose that the origin and the positive $x$ direction have been chosen so that for real values of $x$ the sign of the latter is always that of $\phi^{2}$.

Let $u(x)$ be any solution of the differential equation (2a) which is real over the axis of reals. For negative values of $x$ such that $x \leqq x_{-N}$ this solution is represented asymptotically by a linear combination of the forms (6), that is, with $\xi$ defined as in formula (9),

$$
u(x) \sim \frac{c_{1}}{\phi^{1 / 2}} e^{i \xi}+\frac{c_{2}}{\phi^{1 / 2}} e^{-i \xi} .
$$

For these negative values of $x$ it will be recalled that $\arg \phi^{2}=\pi$ and $\arg \xi=3 \pi / 2$.

In the upper half of the complex $x$ plane let a semi-circle be drawn with center at the origin and with a radius $r$, (i) such that $-r \leqq x_{-N}$ and $r \geqq x_{N}$, (ii) such that at each point of the arc an asymptotic representation (20a) exists, and (iii) such that within the semi-circle the hypothesis made concerning $\phi^{2}$ is fulfilled. As $x$ traverses this semi-circle in the clockwise sense the point $\xi$ in the complex $\xi$ plane traces out a curve $\Gamma$ which begins on the negative axis of imaginaries, proceeds on the whole in the clockwise sense about the point $\xi=0$, and ends on the positive axis of reals. We wish to consider the vicissitudes of the form (20a) during the transit of this arc.

Let it be supposed that the first term of the form (20a) is never dominant on the $\operatorname{arc} \Gamma$. In this case the reality of the solution for the negative values of $x$ requires that $\arg c_{2}=\pi / 4$. The continuity of the solution is not contrary to a suitably moderate change in the coefficient of the submerged term, say from $c_{1}$ to $c_{1}^{*}$, but would be irreconcilable with any change in $c_{2}$. Finally, the reality of the solution for positive values of $x$, where both $\phi^{2}$ and $\xi$ are positive, requires that in the end $c_{1}^{*}$ and $c_{2}$ be complex conjugates. From this the ultimate form, valid for $x \geqq x_{N}$, is deduced to be

$$
u(x) \sim \frac{\left|c_{2}\right|}{\phi^{1 / 2}}\left\{e^{i \xi-i \arg c_{2}}+e^{-i \xi+i \arg c_{2}}\right\} .
$$


This, and the form (20a), give precisely the members of the relation (19a), which is thus established, in the sense that the left hand form implies the right. It will be noted that no deduction of the left hand form from the right by means such as outlined is possible, which accords with the earlier discussion of the relation in question.

The method was applied by $Z$ waan only when the coefficient $c_{1}$ in form (20a) is zero. It is at once evident that in most simple applications this yields an instance of the type considered, and hence affords a means, and a very simple one, for deriving the connection (19a).

The obvious advantage of the method lies in its great simplicity in cases in which it can be applied. Its weaknesses are several. Firstly, there is its excessively circumscribed range. Limited to the case of a simple zero of $\phi^{2}$, there seems no possibility of obtaining from it any result whatever when the zero is of higher order. Secondly, there are involved in it many tacit assumptions, some by no means negligible, particularly as to the asymptotic character of the solutions in the complex plane. A critique and elaboration of this point is contained in the address recently delivered here at Chicago by Birkhoff. $\dagger$ Finally, an estimation of the relative error involved in the result is almost impossible, and unlike either the method of Jeffreys or that which I shall next discuss, the procedure yields no information regarding the functional character of a given solution in the neighborhood of a point where $\phi^{2}$ becomes zero.

9. Another Method. $\ddagger$ The procedures which I have outlined in the two preceding sections are evidently to be characterized as of the nature of means for knitting together the results given by the classical process of $\S 3$ for each of two separate portions, namely $\left(a, x_{-N}\right)$ and $\left(x_{N}, b\right)$, of the fundamental interval $(a, b)$. Under them a solution of the differential equation is thought of as asymptotically fitted in the one sub-interval, likewise so

$\dagger$ G. D. Birkhoff, Quantum mechanics and asymptotic series, this Bulletin, vol. 39 (1933), p. 696.

$\ddagger$ R. E. Langer, On the asymptotic solutions of ordinary differential equations, with an application to the Bessel functions of large order. Transactions of this Society, vol. 33 (1931), p. 23; and On the asymptotic solutions of differential equations, with an application to the Bessel functions of large complex order, Transactions of this Society, vol. 34 (1932), p. 447. 
in the other, and devices are then added for patching the results together into a more or less complete representation. The method which I would now like to present to you attacks the problem from a more fundamental standpoint, that of obtaining at one and the same stroke a representation which is valid over the whole domain of the variable. Should there be no zero of the coefficient $\phi^{2}$ in this domain, then the procedure of itself takes on the classical form. It is a method, therefore, which seeks to include and generalize rather than to extend by piecing out the method heretofore classical. Of its definiteness, adaptability, and scope I shall wish to say something anon.

A scrutiny of the special differential equation (17) and of its explicit solutions (17a) suggests the consideration in the general case of the functions

$$
v_{ \pm} \equiv \xi^{\mu} J_{ \pm \mu}(\xi)
$$

in which $J_{ \pm \mu}$ stands, as customarily, for the Bessel functions of the indicated order, $\mu$ is a tentatively undetermined constant, and $\xi$ is defined in the manner of formula (9). The differential equation satisfied by these functions is

$$
\frac{d^{2} v}{d \xi^{2}}+\frac{(1-2 \mu)}{\xi} \frac{d v}{d \xi}+v=0,
$$

or, in terms of $x$ as the independent variable,

$$
v^{\prime \prime}+\left\{(1-2 \mu) \frac{\lambda \phi}{\xi}-\frac{\phi^{\prime}}{\phi}\right\} v^{\prime}+\lambda^{2} \phi^{2} v=0 .
$$

If in this, finally, the term of the first order is removed, the substitution being

$$
y=\Psi v, \quad \text { with } \quad \Psi=(\xi / \lambda)^{(1 / 2)-\mu} / \phi^{1 / 2},
$$

the resulting equation is found to be

$$
y^{\prime \prime}+\left\{\lambda^{2} \phi^{2}(x)-\frac{\Psi^{\prime \prime}(x)}{\Psi(x)}\right\} y=0,
$$

of which the solutions are accordingly

$$
y_{ \pm}=\Psi(x) \xi^{\mu} J_{ \pm \mu}(\xi)
$$


Superficially the differential equation (21) is of the type of the equation (2) with which we are primarily concerned. That, however, was true also of the equation (10) which is solved by the leading terms of the classical asymptotic forms. The test lies in the functional character of the coefficient $\Psi^{\prime \prime} / \Psi$, which is given explicitly by the formula

$$
-\frac{\Psi^{\prime \prime}}{\Psi}=\frac{\phi^{\prime \prime}}{2 \phi}-\frac{3 \phi^{\prime 2}}{4 \phi^{2}}+\left(\frac{1}{4}-\mu^{2}\right) \frac{\phi^{2}}{\left(\int_{x_{0}}^{x} \phi d x\right)^{2}} .
$$

This evaluation shows that the difference between the equations (22) and (10) consists solely of the presence or absence of the term on the extreme right of (23). Near $x_{0}$, the zero of $\phi^{2}$, the coefficient in equation (10) has a pole, a fact which presages the breakdown of the asymptotic representation as the value $x_{0}$ is approached. The salient fact now is that this pole, which for a random choice of the constant $\mu$ exists also in the function (23), may be obviated in the latter by the choice of $\mu$ as the number $1 /(\nu+2)$, where $\nu$ is the order to which $\phi^{2}(x)$ becomes zero at $x_{0}$. This order may be any non-negative real number. The case $\nu=0$, with the consequent $\mu^{2}=1 / 4$, evidently causes the equation (21) to revert into identity with the equation (10), whereby the inclusion of the theory of $\$ 3$ is at once made evident.

With the indicated choice of $\mu$, the equation (21) yields an approximation to the given equation (2) over an entire interval including the critical point $x_{0}$ provided the coefficient $\phi^{2}$ is otherwise bounded from zero. The nature of this approximation as it differs from that in the method of Jeffreys should be noted. There the difference between the two equations approaches zero, to be sure, at the critical point, but for every other fixed point it increases with $\lambda$ to the order of $\lambda^{2}$. In the present case the difference between the equations is entirely independent of $\lambda$ and is therefore the more effectively overshadowed by the term $\lambda^{2} \phi^{2}$ the larger the value of $\lambda$ is taken.

It was remarked in $\$ 3$ that the permissibility of infinite values for $a$ or $b$, the limits of the interval, was contingent upon the convergerce of the integral (5). The corresponding developments in the present method require the convergence of the integral

$$
\int\left|\frac{x}{\phi}-\frac{\Psi^{\prime \prime}}{\phi \Psi}\right| d x
$$


over intervals not including the critical point $x_{0}$. Since the integrands differ only by the function

$$
\left(\frac{1}{4}-\mu^{2}\right) \frac{\phi d x}{\left(\int_{x_{0}}^{x} \phi d x\right)^{2}},
$$

which integrates directly into $\left(\mu^{2}-1 / 4\right) / \int_{x_{0}}^{x} \phi d x$, and since this approaches a limit as $|x| \rightarrow \infty$, it is clear that the generalized method requires none but the original condition in this respect.

The close resemblance between the differential equations (2) and (21), which becomes the more pronounced as $\lambda$ increases, warrants the presumption that with suitable adjustments the solutions of the latter will represent asymptotically those of the former. I shall sketch out in the next section a mode of proof for this. In doing so I shall, however, content myself here with the consideration of the simplest case, namely with $\lambda$ real and positive, with $\phi^{2}(x)$ real for real values of $x$, with the zero of $\phi^{2}$ simple and located at the origin and with the sign of $\phi^{2}$ the sign of $x$.

10. Proof of the Representation. The differential equation (2) may evidently be written if desired in the form

$$
u^{\prime \prime}+\left\{\lambda^{2} \phi^{2}-\frac{\Psi^{\prime \prime}}{\Psi}\right\} u=\delta(x) \cdot u
$$

with

$$
\delta(x)=\chi(x)-\frac{\Psi^{\prime \prime}(x)}{\Psi(x)} .
$$

For this equation, however, the part of the reduced equation is taken by (21), and on regarding (2*) formally as a non-homogeneous equation, a familiar procedure permits it to be solved in the form

$$
u(x)=y(x)+\frac{1}{W} \int_{c}^{x} H(x, t) u(t) d t
$$

with

$$
H(x, t) \equiv\left\{y_{0}(x) y_{1}(t)-y_{1}(x) y_{0}(t)\right\} \delta(t) .
$$

In this $y_{0}$ and $y_{1}$ may be any independent solutions of the equation (21); $W$ is their Wronskian; $c$ is any point of the interval 
$(a, b)$; and $u(x)$ is the solution of the equation (2) which coincides at $x=c$ with $y(x)$, any chosen solution of (21). I use the term coincides to mean that at the point in question the solutions take the same value, with derivatives which are also equal.

The equation (24) is an integral equation. If the entire right hand member is substituted for $u(t)$ in the integrand, and the process is repeated again and again, etc., there results formally the infinite development

$$
u(x)=y(x)+\frac{1}{W} \int_{c}^{x} H(x, t) y(t) d t
$$

$$
+\frac{1}{W^{2}} \int_{c}^{x} \int_{c}^{s} H(x, s) H(s, t) y(t) d t d s+\cdots .
$$

I will anticipate by mentioning that if $y(x)$ and $c$ are suitably chosen, and $\lambda$ is sufficiently large, this development converges and actually represents $u(x)$.

We are considering the case in which $\phi^{2}(x)$ has a simple zero, that is, in which $\mu=1 / 3$, and among these is included the special equation of $\$ 6$. It will be found that the formula (14) gives the solutions of the equation (21), and without modifications other than such as are implicitly involved in the broader significance of the symbols. The asymptotic forms of these solutions are given by the formulas (16). With the special values $\eta=0$ and $\eta=\pi / 2$, there are obtained thus a pair of solutions which may be used as $y_{0}$ and $y_{1}$ and which are respectively of the forms, when $x$ is negative or positive, indicated by the relations:

$$
\begin{aligned}
& y_{0}(x) \sim \frac{1}{\left.2|\phi|\right|^{1 / 2}} e^{-|\xi|} \sim \frac{1}{\phi^{1 / 2}} \cos \left(\xi-\frac{\pi}{4}\right), \\
& y_{1}(x) \sim \frac{1}{|\phi|^{1 / 2}} e^{|\xi|} \sim \frac{1}{\phi^{1 / 2}} \cos \left(\xi+\frac{\pi}{4}\right) .
\end{aligned}
$$

The Wronskian of this pair has the value $\lambda$.

The first integral term on the right of the relation (24a) is explicitly the sum of the two expressions 


$$
\begin{aligned}
& I_{1}=\frac{y_{0}(x)}{\lambda} \int_{c}^{x} \delta(t) y_{1}(t) y(t) d t, \\
& I_{2}=\frac{-y_{1}(x)}{\lambda} \int_{c}^{x} \delta(t) y_{0}(t) y(t) d t .
\end{aligned}
$$

The points $c$ and $x$ have not as yet been specified. It may be observed, however, that if the range $(c, x)$ contains wholly or partially the interval $\left(x_{-N}, x_{N}\right)$, the integration over this latter contributes to the integral an amount which approaches zero with $1 / \lambda$, for although the product of any two solutions $y(x)$ given by (14) has $\lambda^{1 / 3}$ as a factor, it is otherwise bounded and the interval itself is in length of the order of $\lambda^{-2 / 3}$. The integrations over the range where $|\xi(t)| \leqq N$ may, therefore, be disregarded henceforth. The considerations when $|\xi(t)|>N$ are with advantage shaped to differentiate the cases in which the solution $y(x)$ is rising at $x_{-N}$ from those in which it is falling at that point.

Case 1. $y(x)$ rising on $c<x \leqq x_{-N}$. In this case, as was found in $\$ 6$,

$$
\tan \eta \leqq \frac{1}{2} e^{-2|\xi(c)|},
$$

and from formulas (16)



It is directly clear that when $|\xi(t)|>N$, the integrand in the expression $I_{1}$ is bounded, whence the same follows for the entire integral. The integrand in $I_{2}$, on the other hand, is of the form

$$
\frac{\delta(t)}{\phi(t)} e^{-2 i \xi(t)} O(1),
$$

with $O(1)$ designating a bounded function. Since

$$
\left|e^{-2 i \xi(t)}\right| \leqq\left|e^{-2 i \xi(x)}\right|, \quad \text { whenever } \quad t \leqq x,
$$

it being immaterial whether $t$ or $x$ is positive or negative, the integral in question is seen to be of the form $e^{-2 i \xi(x)} O(1)$. Thus 
the sum $I_{1}+I_{2}$, that is, the second term on the right of $(24 \mathrm{a})$, is concluded to be of the form

$$
\frac{O(1)}{\lambda^{5 / 6}}, \text { when }|\xi| \leqq N \text {, and } \frac{O(1)}{\lambda \phi^{1 / 2}} e^{-i \xi} \text {, when }|\xi|>N \text {. }
$$

This mode of appraisement is extensible in a direct manner to the subsequent terms of (24a) and shows them to be of a similar type with successively higher powers of $\lambda$ in the denominator, sufficient to cause convergence of the series. From this and the form of $y(x)$ it clearly follows that:

If $u(x)$, a solution of equation (2), coincides with a rising solution $y(x)$ of equation (21) at a point $c$, where $c<x_{-N}$, then

$$
\text { (26a) } u(x)=\left\{\begin{array}{lr}
\frac{\cos \eta}{2|\phi(x)| 1 / 2} e^{-|\xi|}\{1+O(1 / \xi)+O(1 / \lambda)\}, & \text { when } c \leqq x \leqq x_{-N}, \\
y(x)+\frac{O(1)}{\lambda^{5 / 6}}, & \text { when } x_{-N} \leqq x \leqq x_{N}, \\
\frac{1}{\phi^{1 / 2}(x)}\left\{\cos \left(\xi-\frac{\pi}{4}+\eta\right)\right. & \text { when } x \geqq x_{N},
\end{array}\right.
$$

the phase constant $\eta$ being that in the representation (25a).

Case 2. $y(x)$ falling at $x_{-N}$. In this case $\eta \geqq \eta_{0}$, with the latter given by $(15 \mathrm{a})$, and

$$
y(x) \sim \begin{cases}\frac{\sin \eta}{|\phi|^{1 / 2}} e^{|\xi|}, & \text { when } x \leqq x_{-N}, \\ \frac{1}{\phi^{1 / 2}} \cos \left(\xi-\frac{\pi}{4}+\eta\right), & \text { when } x \geqq x_{N} .\end{cases}
$$

The integrands in the expressions $I_{1}$ and $I_{2}$ are respectively of the forms

$$
\frac{\delta(t)}{\phi(t)} e^{2 i \xi(t)} O(1), \quad \text { and } \quad O(1)
$$


when $|\xi(t)|>N$, and if $c$ is chosen as any point to the right of $x_{-N}$, the integrals are easily recognized as of the forms

$$
e^{2 i \xi(x)} O(1), \quad \text { and } O(1) \text {. }
$$

The conclusion which follows as in Case 1 is the following:

If $y(x)$, a solution of equation (21), is falling at $x_{-N}$, and if $u(x)$, a solution of equation (2), coincides with $y(x)$ at a point $c$, where $x_{-N} \leqq c$, then

(26b) $u(x)=\left\{\begin{array}{lr}\frac{\sin \eta}{|\phi(x)| 1 / 2} e^{|\xi|}\{1+O(1 / \xi)+O(1 / \lambda)\}, & \text { when } x \leqq x_{-N}, \\ y(x)+\frac{O(1)}{\lambda^{5 / 6}}, & \text { when } x-N \leqq x \leqq x_{N}, \\ \frac{1}{\phi^{1 / 2}(x)}\left\{\cos \left(\xi-\frac{\pi}{4}+\eta\right)\right. & \\ +O(1 / \xi)+O(1 / \lambda)\}, & \text { when } x \geqq x_{N},\end{array}\right.$

with $\eta$ determined by the relations $(25 \mathrm{~b})$.

11. Scope of the Method. The proof which I have thus outlined applied especially to the case of a real parameter and variable and a simple zero of $\phi^{2}$. I would emphasize, however, that these restrictions are in no way demanded by the method, but were imposed here for the sole purpose of avoiding tedious detail in the presentation. Generally the coefficient $\phi^{2}$ may be any function, real or complex, which, in a given region $R_{x}$ of the complex $x$ plane, admits of representation in a form $\left(x-x_{0}\right)^{\nu} \phi_{1}^{2}(x)$, with $\nu$ any non-negative real number and $\phi_{1}{ }^{2}(x)$ an analytic function which is bounded from zero. The parameter $\lambda$ likewise need not be real. When such is the case the formula (9) defines $\xi$ as a complex variable which ranges over a domain $R_{\xi}$ obtainable by mapping from the original region. This domain is in general a portion of a Riemann surface with just one branch point located at $\xi=0$, with sheets in number finite or infinite depending upon the character of the number $\nu$, and with boundaries depending upon $\lambda$ and the boundaries of $R_{x}$. 
The deduction of the approximating differential equation (21) is a formal matter unaffected by the greater or less generality of the elements it involves, and in the domain $R_{\xi}$, the solutions (22) are classically known for all values of $\xi$. As was remarked in $\$ 5$, the asymptotic representations of these solutions are subject to the Stokes' phenomenon, which interposes itself in the transit of any upper or lower half-plane of the Riemann surface $R_{\xi}$. The sub-regions on this surface in which a single representation maintains, correspond to specific sub-regions of the original domain of $x$ which are determinable from the relating map. Since, as in the simple case, the functions $y(x)$ represent asymptotically the properly associated solutions $u(x)$ of the differential equation under consideration, the quantitative details of the Stokes' phenomenon as it applies to the latter are revealed.

The procedure of which the foregoing is an outline has been carried out fully in the instance of several differential equations of classical importance. By suitable transformation, for example, the differential equation of the Bessel functions $J_{\lambda}(z)$ may be given the form of the equation (2) whenever $|\lambda|$ is large, and the structure of these important functions for all complex values of the variable and all large complex indices may therewith be derived. $\uparrow$ The differential equation for the Hermite polynomials is a case of this kind, and a study of the solutions of this equation, or of its transform, the Weber equation, over the entire complex plane and for any large index, real or complex, has been successfully made. $\ddagger$ Lastly, I have made a study by this method of the solutions of the Mathieu equation over the complex plane of the variable and with all relative configurations of the two (real) parameters with one of them large. $\$$ In the instance of several other standard differential equations the method evidently makes a similar study possible.

12. A More General Type of Differential Equation. The function $\chi(x)$ in the coefficient of the equation (2) has throughout the discussion played a role of almost negligible importance. It

$\dagger$ R. E. Langer, loc. cit.

$\ddagger$ N. Schwid, The asymptotic forms of the Hermite and Weber functions, Dissertation, Wisconsin, (1934).

$\S \mathrm{R}$. E. Langer, The solutions of the Mathieu equation with a complex variable and at least one parameter large, Transactions of this Society, vol. 36 (1934). 
will perhaps be granted in view of this that a dependence of this function upon $\lambda$ is not significant so long as it remains bounded with respect to $\lambda$. It can, in fact, be shown that when the zero of $\phi^{2}$ is a simple one the theory requires no essential modification even when $\chi$ is of the order of $\lambda$. That, however, is not so when the zero of $\phi^{2}$ is of higher degree. Only the simplest of such cases, namely that of an equation

$$
u^{\prime \prime}-\left\{\lambda^{2} \phi^{2}(x)+\lambda \theta(x)+\chi(x, \lambda)\right\} u=0,
$$

in which $\phi^{2}$ has a zero of the second order, has to my knowledge been at all investigated. Yet such equations have arisen in a number of connections and those studies which have been made have been made under the spur of impending applications.

Apparently the first discussion of an equation of the type (27) was given by Goldstein $†$ in 1931. His method is an adaptation of that of Jeffreys discussed in $\$ 7$. The variable and parameter are assumed to be real and the term $\chi(x, \lambda)$ does not occur in his equation. In consonance with the basic character of the method it is assumed that near $x_{0}$, the zero of $\phi^{2}$, the functions $\phi^{2}$ and $\theta$ are approximated to the requisite degree of accuracy by $c_{1}\left(x-x_{0}\right)^{2}$ and $c_{2}$, respectively, where $c_{1}$ and $c_{2}$ are constants. The approximating equation,

$$
y^{\prime \prime}-\left\{\lambda^{2} c_{1}\left(x-x_{0}\right)^{2}+\lambda c_{2}\right\} y=0,
$$

may be transformed into a Weber equation and with the solutions of this (which are of classically known forms) playing the role taken in $\$ 7$ by the Bessel functions, the connections between the asymptotic representations which maintain on the two sides of $x_{0}$ are deduced.

The differential equation of this type has presented itself also in quantum mechanics, and in this connection I find a discussion of it to have been given by Voss in 1933. $\ddagger$ There is apparently no essential difference between his treatment and that of Goldstein.

The method of $\$ 9$ likewise lends itself to the generalization

$\dagger \mathrm{S}$. Goldstein, $A$ note on certain approximate solutions of linear differential equations of the second order, (2), Proceedings of the London Mathematical Society, (2), vol. 33 (1932), p. 246.

$\ddagger \mathrm{W}$. Voss, Bedingungen für das Auftreten des Ramsauereffektes, Zeitschrift für Physik, vol. 83 (1933), p. 581. 
necessary for the discussion of the equation (27), and this, moreover, without restriction to real values of the variables. $\dagger$ Since the formulas as stated for the general case are of very considerable complexity, it might be worth noting here the forms which apply when the parameter and variable are real and the former is positive.

The equation is first to be adjusted so that the relation

$$
3 \phi^{\prime}\left(x_{0}\right) \theta^{\prime}\left(x_{0}\right)-2 \phi^{\prime \prime}\left(x_{0}\right) \theta\left(x_{0}\right)=0
$$

is satisfied. Then if $x_{0}$ is taken as the origin, and if $\phi^{2}$ is bounded from zero except at the origin, $\ddagger$ there are solutions describable as follows:

Case 1. $\phi^{2}(x) \geqq 0$ : solutions exponential. With $k$ and $\xi$ defined by the relations

$$
k=\frac{-\theta(0)}{4 \phi^{\prime}(0)}, \quad \xi=\lambda \int_{0}^{x} \phi d x+\int_{0}^{x}\left\{\frac{\theta}{2 \phi}+\frac{k \phi}{\int_{0}^{x} \phi d x}\right\} d x,
$$

there are solutions of the asymptotic forms

$$
u_{\alpha}(x) \sim\left\{\begin{array}{lr}
\frac{(2 \xi)^{k} e^{-\xi}}{\lambda^{1 / 4} \phi^{1 / 2}}, & \text { when } x \geqq x_{N}, \\
\frac{1}{\lambda^{1 / 4}|\phi|^{1 / 2}}\left\{\frac{2 \pi(2 \xi)^{-k} e^{\xi}}{\Gamma(1 / 4-k) \Gamma(3 / 4-k)}-\delta \cdot(2 \xi)^{k} e^{-\xi}\right\}, & \text { when } x \leqq x_{-N},
\end{array}\right.
$$

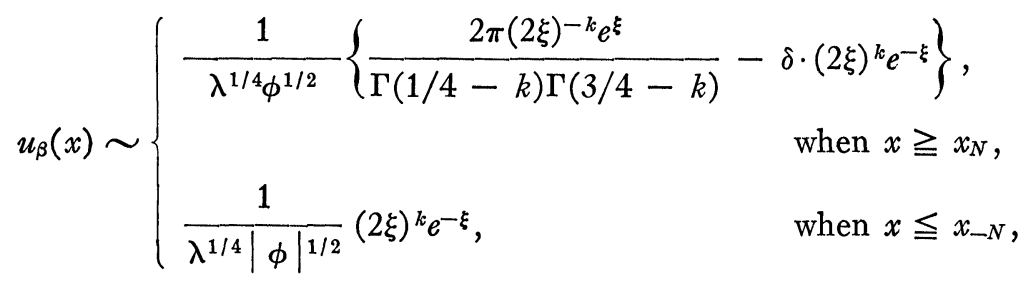

with $\delta=i e^{2 k \pi i}$. It will be noted that $\delta= \pm 1$ when $k=n+1 / 2 \pm 1 / 4$, that is, at the values of $k$ for which the gamma functions become infinite. For such values a third solution which may be used is

$\dagger \mathrm{R}$. E. Langer, The asymptotic solutions of certain linear ordinary differential equations of the second order, Transactions of this Society, vol. 36 (1934), p. 90.

$\ddagger$ For more precise statements I refer to Langer, loc. cit. 


$$
u_{\gamma}(x) \sim \begin{cases}\frac{1}{\lambda^{1 / 4} \phi^{1 / 2}}(2 \xi)^{-k} e^{\xi}, & \text { when } x \geqq x_{N}, \\ \frac{\delta}{\lambda^{1 / 4}|\phi|^{1 / 2}}(2 \xi)^{-k} e^{\xi}, & \text { when } x \leqq x_{-N} .\end{cases}
$$

It is to be understood that arg $\xi$ is to be taken as zero for both positive and negative values of $x$.

Case 2. $\phi^{2}(x) \leqq 0$ : solutions oscillatory. With $k$ and $\xi$ given by the formulas

$$
\begin{aligned}
& k=\frac{\theta(0)}{4\left|\phi^{\prime}(0)\right|}, \\
& \xi=\lambda \int_{0}^{x}|\phi| d x-\int_{0}^{x}\left\{\frac{\theta}{2|\phi|}-\frac{k|\phi|}{\int_{0}^{x}|\phi| d x}\right\} d x,
\end{aligned}
$$

there are solutions which when $\xi$ is small are of the forms

$$
\begin{aligned}
& u_{\delta}(x)=\frac{(2 \xi)^{3 / 4}}{\lambda^{1 / 4}|\phi|^{1 / 2}}\left\{1+\frac{4}{3} k \xi+\cdots\right\}, \\
& u_{\epsilon}(x)=\frac{(2 \xi)^{1 / 4}}{\lambda^{1 / 4}|\phi|^{1 / 2}}\{1+4 k \xi+\cdots\},
\end{aligned}
$$

and which when $\xi$ is large are of the forms

$$
\begin{aligned}
\begin{aligned}
u_{\delta}(x)=\frac{(x /|x|) e^{k \pi / 2}(\pi /|\phi|)^{1 / 2}}{\lambda^{1 / 4}|\Gamma(3 / 4+i k)|} \cos \{\xi & -k \log 2 \xi \\
& \left.+\arg \Gamma(3 / 4+i k)-\frac{3 \pi}{8}\right\} \\
u_{\gamma}(x)=\frac{2 e^{k \pi / 2}(\pi /|\phi|)^{1 / 2}}{\lambda^{1 / 4}|\Gamma(1 / 4+i k)|} \cos \{\xi-k \log 2 \xi & \left.+\arg \Gamma(1 / 4+i k)-\frac{\pi}{8}\right\} .
\end{aligned}
\end{aligned}
$$

The uncertainty or vagueness of these formulas is of the order of $1 / \xi$ and of $(\log \lambda) / \lambda$.

13. Remarks on the Wave Equation. The Schrödinger wave equation for a particle with one degree of freedom is familiarly of the form 


$$
u^{\prime \prime}+\frac{8 \pi^{2} m}{h^{2}}\{E-V(x)\} u=0
$$

with $m$ the mass of the particle, $E$ and $V(x)$, respectively, the total energy and the potential energy, and $h$ denoting Planck's constant. A wave function is a solution of the equation which remains bounded over the entire infinite axis of $x$. The cases of especial interest are those in which $E-V(x)$ changes sign for one or more values of $x$, and when such is the case the equation is evidently one of essentially the type we have been considering. In classical mechanics motion is possible only when $E-V(x)$ is positive. These are the intervals in which the solutions of the equation (28) are oscillatory. A wave function, that is, a permanently bounded solution, will in general exist only for special values of $E$, called characteristic values, the determination of which generally constitutes the essential part of the problem.

It is customary in the literature of the equation (28) to treat it as of the type of the equation (2) with the roles of $\lambda^{2}$ and $\phi^{2}$ taken respectively by the expressions $4 \pi^{2} / h^{2}$ and $2 m\{E-V(x)\}$, and with $\chi \equiv 0$. The "W.K.B." procedure is then applied to supply the connection formulas across a zero of $\phi^{2}$ for the representations of $u(x)$ asymptotic with respect to $\lambda$. In these representations the leading terms alone are generally considered. There are in this process a number of points to which I should like to call attention since, if nothing more, they hold the potentialities of error, and in the disregard of which conclusions have in a number of instances been drawn which seem to lack complete justification.

Firstly, the value assigned to $\lambda$, though in the prevailing units it may be large, is nevertheless fixed. The designation of a quantity as "of the order of $1 / \lambda$ " is therefore not a significant one, and formulas asymptotic with respect to $\lambda$ should be used only with caution. It would seem that when such formulas are employed an actual verification that the relative vagueness of the formula is within the allowable margin of error is indispensable, and that this is particularly so when conclusions concerning quantities which may be relatively small are involved. Secondly, the function $2 m\{E-V(x)\}$ is dependent upon the parameter $E$. In this respect it differs from the $\phi^{2}$ of the general asymptotic theory. When the range of values of $E$ is considera- 
ble and also when $E$ lies in certain critical ranges, this feature is easily conceived to be, and may be shown to be, one of first importance. Thirdly, the points at which the solutions change in character from the exponential to the oscillatory are not fixed but vary with the parameter $E$, a matter which is demonstrably not to be lightly ignored. For if, in particular, two such points approach each other too closely, the representations ordinarily obtained become entirely invalid.

Having raised these issues I would like to present a variation of the procedure outlined which seems to me to be more revealing, and logically much more defensible, though from the purely manipulative standpoint it is practically identical with the customary one. For convenience, I shall think of the case in which the function $2 m\{E-V(x)\}$ is positive on a single finite interval, say $x_{0} \leqq x \leqq x_{0}^{\prime}$. The substitution

$$
s=\frac{x-x_{0}}{x_{0}^{\prime}-x_{0}}
$$

gives to the equation (28) the form

$$
\frac{d^{2} u}{d s^{2}}+\lambda^{2} \phi^{2}(E, s) u=0
$$

in which we may choose

$$
\begin{aligned}
\lambda & =\frac{2 \pi}{h} \int_{x_{0}}^{x_{0}{ }^{\prime}}\{2 m(E-V(x))\}^{1 / 2} \cdot d x, \\
\phi(E, s) & \equiv \frac{\{E-V(s)\}^{1 / 2}}{\int_{0}^{1}\{E-V(s)\}^{1 / 2} d s} .
\end{aligned}
$$

In this form almost all the questions raised above have been obviated. In the first place, $\lambda$ is not fixed but increases with $E$. Secondly, though, to be sure, $\phi^{2}$ still depends upon $E$, this dependence has been normalized, so to speak ironed out, to the extent that the area under the graph of $\phi$ between its zeros is fixed at the value 1 . Thirdly, the zeros of $\phi^{2}$ are fixed, specifically at $s=0$ and $s=1$. Evidently the equation in the form (29) differs in only a very minor way from the equation (2) of the general theory. Indeed, in the particular example $V(x) \equiv x^{2}$ which is often invoked, the equation is 


$$
\frac{d^{2} u}{d s^{2}}+\frac{128 \pi^{2} m E^{2}}{h^{2}}\left(s-s^{2}\right) u=0,
$$

which is precisely of the type considered.

From the form (29) the determination of the characteristic values proceeds in the usual way. The solution which is bounded on the range $-\infty<s<0$, must be that given by (26a) with $\eta=0$. Its form in a fixed interval entirely within $(0,1)$ must, therefore, be

$$
\frac{1}{\phi^{1 / 2}(s)}\left\{\cos \left(\xi-\frac{\pi}{4}\right)+O(1 / \lambda)\right\}
$$

with $\xi=\lambda \int_{0}^{s} \phi d s$. Similar considerations, with the origin replaced by the point $s=1$, and the direction of the axis reversed, show that the solution bounded on $1<s<\infty$ must in the oscillatory interval have the form

$$
\frac{ \pm 1}{\phi^{1 / 2}(s)}\left\{\cos \left(\xi_{1}-\frac{\pi}{4}\right)+O(1 / \lambda)\right\},
$$

with $\xi_{1}=\lambda \int_{s}^{1} \phi d s$. Since $\xi+\xi_{1}=\lambda$, we may write $(30 \mathrm{~b})$ in the form

$$
\frac{1}{\phi^{1 / 2}(s)}\left\{\cos \left(\xi-\lambda-\frac{\pi}{4} \pm \frac{\pi}{2}\right)+O(1 / \lambda)\right\},
$$

and the condition that this be identical with (30a) is obviously that

$$
\lambda=\left(n+\frac{1}{2}\right) \pi+O\left(\frac{1}{\lambda}\right) .
$$

Since $\lambda$ must be large, this shows that the order of $1 / \lambda$ is the order of $1 / n$, and on substituting the value of $\lambda$ the condition becomes

$$
\frac{2}{h} \int_{x_{0}}^{x_{0}{ }^{\prime}}\{2 m(E-V(x))\}^{1 / 2} d x=n+\frac{1}{2}+O\left(\frac{1}{n}\right) .
$$

In order that a high degree of precision be assured, it is evidently requisite that $n$ be sufficiently large.

If $n$ is only moderate the result, at least without further considerations, is of little value. It may in this case be useful to vary 
the method by skipping over the interval $\left(x_{0}, x_{0}{ }^{\prime}\right)$ entirely in the following way. Since the left member of (31) is not large, the value of $\theta$ given by the formula

$$
\theta=\frac{2 \pi}{h}(2 m)^{1 / 2}\left\{V_{m}-E\right\}
$$

with $V_{m}$ denoting the minimum of $V(x)$, is of moderate magnitude. The equation (28) may, therefore, be written in the form

$$
u^{\prime \prime}-\left\{\lambda^{2} \phi^{2}(x)+\lambda \theta\right\} u=0,
$$

with

$$
\lambda^{2}=\frac{8 \pi^{2} m}{h^{2}}, \quad \phi^{2}=\left\{V(x)-V_{m}\right\}
$$

and is thus reduced to one of the type referred to in $\$ 12$.

The case in which the function $\{E-V(x)\}$ is positive on two or more distinct intervals has also important applications and has received considerable attention in the physical literature. $\dagger$ It would appear, however, that the considerations involved in it call for peculiar caution with respect to the limitations and implications of asymptotic representations, and these it seems have been all too often overlooked.

Let me suppose, to be concrete, that an equation (2) (or (29)) has a coefficient $\phi^{2}$ which is negative on just one interval, say $x_{1}<x<x_{0}$. On this interval the solutions are then of exponential type and in particular there are solutions of each of the forms

$$
\begin{aligned}
& \text { (a) } \frac{1}{|\phi|^{1 / 2}} e^{\left|\xi_{0}\right|}, \\
& \text { (b) } \frac{1}{|\phi|^{1 / 2}} e^{-\left|\xi_{0}\right|}, \quad \text { with } \quad \xi_{0}=\lambda \int_{x_{0}}^{x} \phi d x .
\end{aligned}
$$

$\dagger$ See, for example, D. M. Dennison, and G. E. Uhlenbeck, The two-minima problem and the ammonia molecule, Physical Review, vol. 41 (1932), p. 313; H. D. Koenig, Calculation of characteristic values for periodic potentials, Physical Review, vol. 44 (1933), p. 657; Ta-You Wu, Characteristic values of the two minima problem and quantum defects of states of heavy atoms, Physical Review, vol. 44 (1933), p. 727. 
It may not be concluded from this, however, that the solution represented by (32a) has the form

$$
\frac{1}{\phi^{1 / 2}} \cos \left(\xi_{0}+\frac{\pi}{4}\right),
$$

for $x>x_{0}$, in virtue of the connection formula (19c), for that formula, as was remarked earlier, permits no implication from left to right. In fact, by (26b), the form beyond $x_{0}$ may be

$$
\frac{\csc \eta}{\phi^{1 / 2}} \cos \left(\xi_{0}-\frac{\pi}{4}+\eta\right),
$$

with any $\eta$ exceeding a small value $\eta_{0}$ (given by $15 \mathrm{a}$ ). The form (31b) also implies strictly no more than the form

$$
\frac{2 \sec \eta_{1}}{\phi^{1 / 2}} \cos \left(\xi_{0}-\frac{\pi}{4}+\eta_{1}\right) .
$$

Since the value of $\eta_{1}$ in this is, however, restricted to be so small as to lie below the vagueness which has already been neglected in using only the leading term, the order of the error is not actually increased by suppressing $\eta_{1}$ entirely and writing

$$
\frac{2}{\phi^{1 / 2}} \cos \left(\xi_{0}-\frac{\pi}{4}\right),
$$

which is in accord with the connection formula (19a). With this understanding the form (32b*) may be associated with any solution which is a rising exponential as it approaches the critical point. A situation similar to that at $x_{0}$ is, of course, also confronted in this matter at the point $x_{1}$.

The difficulty here seems to me to be circumventable only by picking special solutions in a suitable way, and holding fast to them throughout the discussion. I will try to make this clear as follows. Let $u_{1}(x)$ be chosen as that solution which for $x<x_{1}$ is of the form

$$
\frac{1}{\phi^{1 / 2}} \cos \left(\lambda \int_{x}^{x_{1}} \phi d x+\frac{\pi}{4}\right) .
$$

It may be implied, for instance, by (26b) that for $x>x_{1}$ the form is 
$\frac{1}{|\phi|^{1 / 2}} e^{\lambda \int_{x_{1}}^{x}|\phi| d x}, \quad$ that is, $\quad e^{\lambda \int_{x_{1}}^{x_{0}}|\phi| d x} \cdot \frac{1}{|\phi|^{1 / 2}} e^{-\lambda \int_{x}^{x_{0}}|\phi| d x}$.

This solution as $x_{0}$ is approached from the left is rising. Hence its form for $x>x_{0}$ may be inferred, as in the case of (32b), and the complete relation indicated thus

$$
\begin{aligned}
& \frac{1}{\phi^{1 / 2}} \cos \left(\lambda \int_{x}^{x_{1}} \phi d x\right.\left.+\frac{\pi}{4}\right) \underset{\text { at } x_{1}}{\longrightarrow} \frac{1}{|\phi| 1 / 2} e^{\lambda \int_{x_{1}}^{x}|\phi| d x} \\
& \underset{\text { at } x_{0}}{\longrightarrow} e^{\lambda \int_{x_{1}}^{x_{0}}|\phi| d x} \cdot \frac{2}{\phi^{1 / 2}} \cos \left(\lambda \int_{x_{0}}^{x} \phi d x-\frac{\pi}{4}\right) .
\end{aligned}
$$

The inference has in each case been in the direction of the arrow.

For a second solution $u_{2}(x)$ we must now choose in the region $x>x_{0}$. In particular, the choice may be placed upon that one of the form

$$
\frac{1}{\phi^{1 / 2}} \cos \left(\lambda \int_{x_{0}}^{x} \phi d x+\frac{\pi}{4}\right)
$$

which then is certainly of the form

$$
\frac{1}{|\phi|^{1 / 2}} e^{\lambda \int_{x}^{x}|\phi| d x}, \quad \text { that is, } e^{\lambda \int_{x}^{x}|\phi| d x} \cdot \frac{1}{|\phi|^{1 / 2}} e^{-\lambda \int_{x_{1}}^{x}|\phi| d x},
$$

when $x<x_{0}$. This, however, is rising as $x$ approaches $x_{1}$ from the right, and hence for $x<x_{1}$ its form is as indicated in the formula

$$
\begin{aligned}
& e^{\lambda \int_{x_{1}}^{x_{0}}|\phi| d x} \cdot \frac{2}{\phi^{1 / 2}} \cos \left(\lambda \int_{x}^{x_{1}} \phi d x-\frac{\pi}{4}\right) \underset{\text { at } x_{3}}{\longleftarrow} \\
& \frac{1}{|\phi|^{1 / 2}} e^{\lambda \int_{x}^{x_{0}}|\phi| d x} \underset{\text { at } x_{0}}{\longleftarrow} \cdot \frac{1}{\phi^{1 / 2}} \cos \left(\lambda \int_{x_{0}}^{x} \phi d x+\frac{\pi}{4}\right),
\end{aligned}
$$

the arrows again showing the direction in which the inference was made.

With a pair of solutions $u_{1}(x), u_{2}(x)$ thus chosen, every solution is, of course, expressible as a combination

$$
u(x)=A u_{1}(x)+B u_{2}(x) .
$$

It is then given asymptotically in each of the three intervals by 
the same combination of forms. In particular, in the interval $x>x_{0}$ we should have

$u \sim \frac{1}{\phi^{1 / 2}}\left\{C \cos \left(\lambda \int_{x_{0}}^{x} \phi d x-\frac{\pi}{4}\right)+B \cos \left(\lambda \int_{x_{0}} \phi d x+\frac{\pi}{4}\right)\right\}$,

with

$$
C=2 A e^{\lambda \int_{x_{1}}^{x_{0}}|\phi| d x},
$$

or, as it may be alternatively written,

$$
u \sim\left\{\frac{B^{2}+C^{2}}{\phi}\right\}^{1 / 2} \cos \left(\lambda \int_{x_{0}}^{x} \phi d x-\frac{\pi}{4}+\tan ^{-1} \frac{B}{C}\right) .
$$

I have carried the formula to this last stage especially to warn against deductions from it which are not justified. For example, to conclude from it that the phase of $u$ differs from that of $u_{1}$ by the amount $\tan ^{-1}(B / C)$ would be warranted and meaningful only if this amount is large enough to project beyond the margin of vagueness which is inherent in all the formulas used. Though this is ignored when only the leading terms are written, it must nevertheless be borne in mind whenever conclusions are being drawn.

The UNIVERSITY OF Wisconsin 